\title{
Pain Around Elbow: Not Always Tennis Elbow!
}

\author{
Hüseyin Sina COŞKUN ${ }^{1, *} \oplus$, Nevzat DABAK ${ }^{1 \oplus}$, Ferhat SAY $^{1} \oplus$, Hasan GÖÇER ${ }^{1 \oplus}$ \\ ${ }^{1}$ Department of Orthopedics and Traumatology, Faculty of Medicine, Ondokuz Mayıs University, Samsun Turkey
}

\begin{abstract}
\begin{tabular}{ccccc}
\hline Received: $12.05 .2020 \quad \bullet \quad$ Accepted/Published Online: 06.12 .2020 & $\bullet$ & Final Version: 14.03 .2021 \\
\hline
\end{tabular}
Abstract

Elbow is a well-known body part of muscular strains and overuse syndromes. Patients presented to the orthopedic department with elbow pain or swelling often diagnosed with benign conditions such as tennis elbow, golfer's elbow, or olecranon bursitis. It must be kept in mind that several tumoral conditions can be seen around the elbow and the patient must be carefully evaluated. In this study, we aimed to demonstrate rare cases of bone or soft tissue tumors around the elbow. Between 2004 and 2017, patients with a symptom of elbow pain or swelling are included in the study. Finally, the study consists of 53 patients. 20 patients were male and 33 were female. The mean age of the patients was $42.94 \pm 15.3$. There were 9 bone tumors, 16 soft tissue tumors, 9 non-classified benign cystic lesions, 3 metastasis, and 16 cases were non-tumoral conditions. 7 of 9 bone tumors were benign. The solitary bone cyst was the most common bone tumor seen around the elbow. 2 malignant bone tumor cases were diagnosed as Ewing Sarcoma and metastatic bone disease. Among the 16 soft tissue tumors, schwannomas are the most presented cases in the tumor council followed by lipoma (6 cases). Even in a multidisciplinary bone and soft tissue tumor council, almost one-third of the elbow pain patients were non-tumoral and the most commonly seen condition was myositis ossificans ( 6 cases), followed by infection or bursitis ( 5 cases). Elbow pain is commonly seen and evaluated by every orthopedic surgeon on outpatient's clinic. It must be kept in mind that tumoral conditions around the elbow are rarely seen but deserve further examination in the presence of chronic pain.
\end{abstract}

Keywords: bone tumor, elbow pain, lateral epicondylitis

\section{Introduction}

Bone and soft tissue tumors can be seen at any age and settle in different localizations of the body. While some tumors are found in characteristic localizations, a wide differential diagnosis list should be established for some tumors. Changes secondary to infection and trauma should be considered in the differential diagnosis of musculoskeletal tumors. An experienced musculoskeletal system oncologist takes into account typical sites of tumors during this diagnostic process (Church et al., 2017).

Elbow is not a common localization for musculoskeletal tumors. Bone tumors around the elbow are rare and its incidence is about 1\% (Morrey and Sanchez-Sotelo, 2009). Literature information on primary bone tumors of the elbow is rare and is only two case series consisting of 75 patients and 25 patients, respectively. (Bruguera and Newman, 1998; Halai et al., 2015). In recent years, progress has been made in the diagnosis, treatment, and prognosis of patients with bone and soft tissue tumors around the elbow. Early diagnosis and preoperative planning are important and can significantly improve the prognosis of these patients (Savvidou et al., 2019).

Non-tumoral causes must primarily be considered in a patient presenting with pathology around elbow joint. One of the most common pathologies in this region is lateral epicondylitis, which is an overuse disorder, also known as the tennis elbow. Patients most often complain of pain in or around the bone protrusion of the lateral epicondyle that spreads in the direction of the diffuse extensor muscle mass in the forearm and sometimes the forearm proximally. From a mild and constant pain to pain that affects all daily activities, a different clinical picture prevails, and in some cases, even night pain can be observed (Vaquero-Picado et al., 2016).

In this article, it is aimed to draw attention to the muscular and skeletal system tumors seen around the elbow and to ensure that the physician does not ignore the muscular and skeletal system tumors in the differential diagnosis in a condition around the elbow. 


\section{Material and method}

Between 2004 and 2017, the database of patients enrolled in a multidisciplinary bone and soft tissue tumor council at Ondokuz Mayis University hospital was retrospectively scanned and transferred to a computer environment. This study included patients of a multidisciplinary bone and soft tissue tumor council evaluated for pain and swelling in the elbow. Patients who normally applied to the orthopedic clinic because of elbow pain were not included in this study. Therefore, the number of patients with suspected elbow tumor evaluated by the tumor council remained lower than the patients who presented to the daily orthopedic outpatient clinic. Among the patients of the tumor council, patients with a symptom of elbow pain or swelling are included in the study. Finally, the study consists of 53 patients. 20 patients were male and 33 were female. The region covering $1 / 3$ distal of the humerus and the proximal ulna and $1 / 3$ of the proximal radius is defined as the elbow and this anatomical location is taken as a criterion. Any patient with swelling and mass in the elbow area can be referred to the tumor council with a preliminary diagnosis of a tumor or soft tissue tumor after being evaluated by an experienced musculoskeletal oncologist. In the council, the clinical images and radiological evaluations of the patient are discussed with multidisciplinary participation and clinical manifestations and imaging studies are discussed. Bone and soft tissue tumors in our article were both diagnosed radiologically and pathologically. Non-tumoral causes were clinically classified again after multidisciplinary conditions evaluation.

The mean age of the patients was $42.94 \pm 15.3$. Data obtained in the study were analyzed statistically using SPSS for Windows 21.0 software (SPSS Inc, Chicago, IL, USA). Descriptive statistics were presented as mean \pm standard deviation, median (minimum-maximum) values, frequency (n), and percentage $(\%)$.

This study was found ethically appropriate by the Ondokuz Mayıs University Clinical Research Ethics Committee on 20.07.2017 with the decision number OMUKAEK 2017/259. No funding was used for the study.

\section{Results}

There were 9 bone tumors, 16 soft tissue tumors, 9 nonclassified benign cystic lesions, 3 metastasis, and 16 cases were non-tumoral conditions. 7 of 9 bone tumors were benign. Giant cell tumor ( 1 case), solitary bone cysts (3 cases), aneurysmal bone cyst (1), and enchondroma (1) are the benign bone tumors seen around the elbow. 2 malignant bone tumor cases were diagnosed as Ewing Sarcoma and metastatic bone disease. Among the 16 soft tissue tumors, they were 3 lipomas, 6 schwannomas, 1 Merkel cell carcinoma, 2 squamous cell carcinoma, 1 myxoid liposarcoma, and 1 undifferentiated pleomorphic sarcoma and 2 metastasis cases. Even in a multidisciplinary bone and soft tissue tumor council, almost one-third of the elbow pain patients were non-tumoral and the most commonly seen condition was myositis ossificans (6 cases), followed by infection or bursitis (5 cases). In our Ewing Sarcoma case, the patient was 15 years old and treatment was started immediately after the biopsy since she applied with a preliminary diagnosis of the mass from a regional hospital (Fig. 1 and 2).

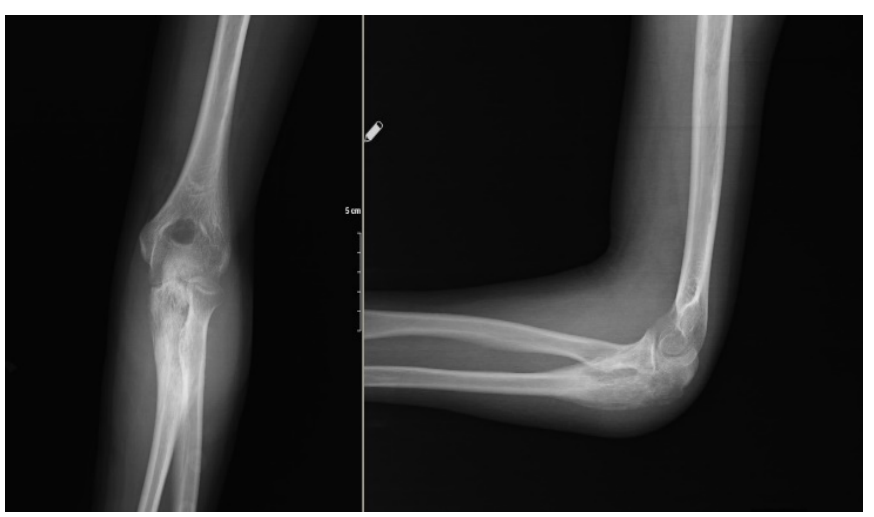

Fig.1. Preoperative direct radiograph of a 15 years-old-female reveals a lytic and expansile lesion around the olecranon

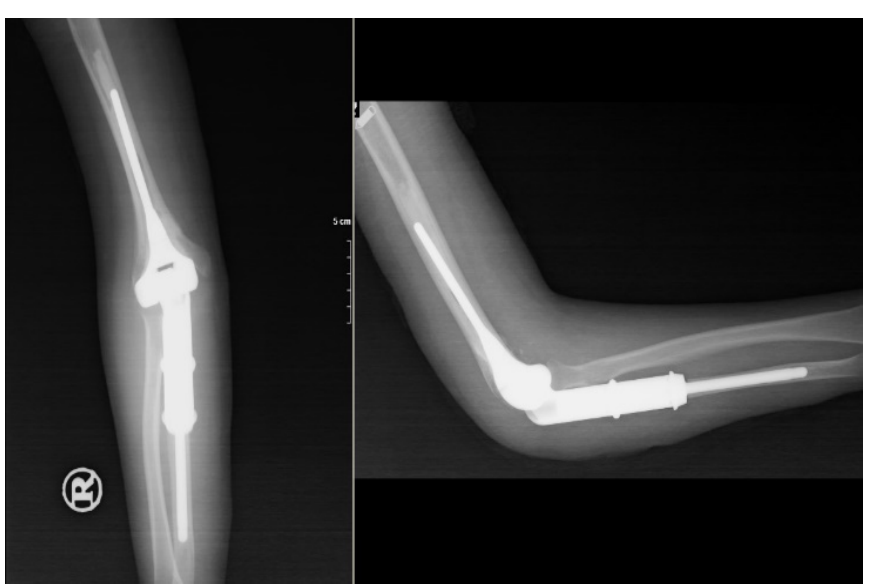

Fig. 2. Postoperative direct radiograph of a 15 years-old-female diagnosed with Ewing Sarcoma, treated by proximal ulnar resection and modular elbow tumor prosthesis. Ulnar nerve and other neurovascular structures have been protected during the surgery and no neurovascular deficit has been noticed postoperatively

Table 1. Diagnosis distribution of patients who referred to the tumor council with swelling and pain around the elbow

\begin{tabular}{|l|c|c|}
\hline Elbow Pain or Swelling & $(\mathbf{n}=\mathbf{5 3})$ & $\mathbf{\%}$ \\
\hline Non-Tumoral Conditions & 16 & 30.18 \\
\hline Soft Tissue Tumors & 16 & 30.18 \\
\hline Benign Cystic Lesions & 9 & 16.98 \\
\hline Bone Tumors & 9 & 16.98 \\
\hline Metastasis Cases & 3 & 5.66 \\
\hline
\end{tabular}

Table 2. Distribution of bone tumors around the elbow

\begin{tabular}{|l|c|}
\hline Bone Tumors & $\mathbf{n = 9}$ \\
\hline Solitary Bone Cyst & 3 \\
\hline Giant Cell Tumor & 1 \\
\hline Aneurysmal Bone Cyst & 1 \\
\hline Enchondroma & 1 \\
\hline Osteochondroma & 1 \\
\hline Metastasis & 1 \\
\hline Ewing Sarcoma & 1 \\
\hline
\end{tabular}


Table 3. Distribution of soft tissue tumors around the elbow

\begin{tabular}{|l|l|}
\hline Soft Tissue Tumors & $\mathbf{n = 1 6}$ \\
\hline Schwannoma & 6 \\
\hline Lipoma & 3 \\
\hline Squamous Cell Carcinoma & 2 \\
\hline Metastasis & 2 \\
\hline Merkel Cell Carcinoma & 1 \\
\hline Myxoid Liposarcoma & 1 \\
\hline Undifferentiated Pleomorphic Sarcoma & 1 \\
\hline
\end{tabular}

Table 4. Distribution of non-tumoral conditions around elbow

\begin{tabular}{|l|c|}
\hline Soft Tissue Tumors & $\mathbf{n = 1 6}$ \\
\hline Myositis Ossificans & 6 \\
\hline Infection or Bursitis & 5 \\
\hline Lateral Epicondylitis & 5 \\
\hline
\end{tabular}

\section{Discussion}

Elbow pain is a common symptom in orthopedics and traumatology outpatient clinics. The examination firstly starts with inspection and if there is a noticeable mass, further evaluation is carried out in this direction. However, often a noticeable change is not detected at first examination. Pathologies around the elbow can be caused by any component of the joint, including the tendon, bursa, bone or nerves. Elbow pain is sometimes manifested by a decrease in grip strength at the level of the wrist. Lateral epicondyle, which is the common starting point of wrist extensor muscles, develops enthesitis due to repetitive trauma or overuse in time. Mostly, lateral epicondylitis can be easily diagnosed without the need for direct radiography. In our series, the number of lateral epicondylitis was quantitatively low since it was referred to as the tumor council and was evaluated among nontumoral causes. However, we know that the incidence in society is higher than in tumors. Patients with a lateral epicondylitis must be carefully evaluated for the presence of other pathologic conditions.

Lateral epicondylitis was first described in 1873 by Runge in the medical literature (Runge, 1873). Rather than an inflammatory condition, it is a chronic symptomatic degeneration that affects the attachment muscles of the forearm to the lateral condyle of the humerus. (Verhaar, 1994). Lateral epicondylitis or 'tennis elbow' is one of the primary admissions to orthopedics and traumatology outpatient clinic due to elbow pain and is a common condition affecting patients. It usually occurs between the ages of 35-55. Often self-limited, but the disease sometimes continues to cause persistent symptoms that may be resistant to treatment in some patients (Ahmad et al., 2013). Patients often complain of pain in or around the bone. Analgesic support and conservative treatment are generally sufficient for its treatment. It is a benign condition that often improves without complications. Since the disease is considered as an easy condition to treat and one of the most common pathology of elbow in daily orthopedic outpatient practice, other pathologies such as bone and soft tissue tumors must be eliminated before starting the treatment of the lateral epicondylitis.

Fortunately, bone tumors that should be considered in patients presenting with elbow pain are less frequently seen compared to lateral epicondylitis. Even today, diagnostic dilemmas and delays in diagnosis are common. Thanks to the advanced imaging techniques, the management and prognosis of patients with elbow bone tumors have improved significantly in recent years. (Savvidou et al., 2019). Even today, the incidence of misdiagnosis is common in up to $13 \%$, often due to the rare occurrence of these lesions, atypical clinical presentation, and low tumoral suspicion. (Halai et al., 2015). Additional imaging and diagnostic studies should be performed in patients with bone lysis, cortical erosion, new bone formation, mineralization, or periosteal reaction on direct radiographs of the elbow. (Miller, 2008).

Ewing sarcoma, a malignant bone tumor, is generally observed in the pediatric age group, in the diaphysis of long bones. It is the second most common malignant bone tumor in terms of incidence in children and adolescents. Its incidence is high in the first 2 decades. In terms of localization, primary malignant bone tumors around ulna are extremely rare. The main malignant bone tumors seen around the elbow are osteosarcoma, primary giant cell bone tumor, and Ewing sarcoma. (Wang and Lin, 2015). In our study, we presented a case of Ewing Sarcoma of 15-year-old female (Fig. 1 and 2).

According to the Leeds region tumor records, the primary tumors of the elbow between 1958 and 1992 found 25 of 2154 cases $(1.2 \%)$. Fifty percent of the cases were localized in the ulna, $20 \%$ in the radius, $20 \%$ in the humerus, and $10 \%$ in the soft tissue. Benign tumors were more common than malignant ones (19-6). While malignant tumors were observed in elderly patients (mean age: 45 years), patients with benign tumors (mean age: 17 years) were observed earlier in life. Preoperative diagnosis was made clinically or radiologically in only 16 patients, and in one case the diagnosis at the time of the first presentation of the disease was changed by the local bone tumor council. Given the difficulty of accurately diagnosing primary bone tumors, all such lesions should be directed to a regional bone and soft tissue tumor council. (Bruguera and Newman, 1998). The number of elbow tumors in our case was 53 and constituted $2.21 \%$ of all bone and soft tissue tumor council cases (a total of 2397 cases).

In our study, the most common bone tumor seen around the elbow was found to be a solitary bone cyst. In the current literature, there are case studies reporting that aneurysmal bone cyst is observed more frequently than a solitary bone cyst around the elbow. (Ozger et al., 2012; Chhawra et al., 2019). An aneurysmal bone cyst is a non-neoplastic, expansile, and osteolytic condition which better be known as a tumor-like condition. It is benign but is evaluated in the local aggressive bone tumor group, and its cause is still unclear. (Mascard et al., 2015). In a study by Chhawra et al., in a 40-year-old male patient, the pathological diagnosis was detected as an 
aneurysmal bone cyst as a result of the pathological fracture in the radial head, which is thought to be secondary to the trauma and tuberculosis disease. The treatment of the patient was wide resection of the affected area and adjuvant phenol was applied after the surgery. In this case, it was pointed out that aneurysmal bone cyst can also be seen in the epiphyseal location. (Chhawra et al., 2019).

In the case of Özger et al., a 13-year-old female patient, aneurysmal bone cyst observed in the coronoid process of the ulna was treated with phenol and cement as an adjuvant treatment following curettage. (Ozger et al., 2012).

In our study, three cases of metastasis were reported around the elbow. All 3 cases were located on the distal humerus. They were patients with multiple metastases of unknown primary foci. Tunio et al. shared a case reporting the metastasis on distal humerus, one year after oncological treatment of a very rare small cell bladder carcinoma. (Tunio et al., 2013). We can explain that elbow metastases are not commonly seen in the literature since these patients usually have multiple metastases and their survival rate is poor.

Schwannoma cases around the elbow have been reported in the literature. (Mariottini et al., 2011; Perrotta et al., 2011). In our study, the most common soft tissue tumor around the elbow was detected as schwannoma. It can be explained by the fact that lipoma, which is a more common soft tissue tumor, is less common than schwannoma, the lipoma cases are relatively easier to recognize, and therefore lipoma cases are presented to the council less frequently.

In conclusion, elbow pain is frequently seen and evaluated by every orthopedic surgeon in outpatient clinics. It should be remembered that tumoral formations around the elbow are rarely seen, but in the presence of chronic pain, further examinations are required. It should be kept in mind that bone tumors can be seen around the elbow, especially in the ulna.

\section{Acknowledgment}

None

\section{Conflict of Interest}

None

\section{References}

1. Ahmad, Z., Siddiqui, N., Malik, S.S., Abdus-Samee, M., Tytherleigh-Strong, G., Rushton, N., 2013. Lateral epicondylitis: a review of pathology and management. Bone. Joint. J. 95-B, 1158-64.

2. Bruguera, J.A., Newman, R.J., 1998. Primary tumors of the elbow: a review of the Leeds Regional Bone Tumour Registry. Orthopedics. 21, 551-3.

3. Chhawra, S., Jain, R., Aggarawal, R., Pandey, A., 2019. A Rare Case of Radius Head Epiphyseal Aneurysmal Bone Cyst with Predisposing Factor as Trauma Tuberculosis of Elbow apart from Genetic. J. Orthop. Case. Rep. 9, 23-27.

4. Church, D.J., Krumme, J., Kotwal, S., 2017. Evaluating SoftTissue Lumps and Bumps. Mo. Med. 114, 289-294.
5. Halai, M., Gupta, S., Spence, S., Wallace, D., Rymaszewski, L., Mahendra, A., 2015. Primary osseous tumours of the elbow: 60 years of registry experience. Shoulder Elbow. 7, 272-81.

6. Mariottini, A., Carangelo, B., Peri, G., Tacchini, D., Mormouras, V., Muya, M., Palma, L., Zalaffi, A., 2011. Schwannoma of median nerve at the elbow. Case report and short review of the literature. G. Chir. 32, 55-8.

7. Mascard, E., Gomez-Brouchet, A., Lambot, K., 2015. Bone cysts: unicameral and aneurysmal bone cyst. Orthop. Traumatol. Surg. Res. 101, S119-27.

8. Miller, T.T., 2008. Bone tumors and tumorlike conditions: analysis with conventional radiography. Radiology. 246, 662-74.

9. Morrey, B.F., Sanchez-Sotelo, J., 2009. The elbow and its disorders. Philadelphia, PA: Saunders, Vol., Elsevier.

10. Ozger, H., Akgül, T., Yıldız, F., Sağlam, Y., 2012. Unusual localization of an aneurysmal bone cyst in ulnar coronoid process. Acta. Orthop. Traumatol. Turc. 46, 144-7.

11. Perrotta, R., Virzì, D., Tarico, M.S., Napoli, P., 2011. An unusual case of symptomatic Schwannoma on the elbow. Br. J. Neurosurg. 25, 306-7.

12. Runge, F., 1873. Zur genese und behandlung des schreibekrampfes. Berl. Klin. Wochenschr. 10, 245-248.

13. Savvidou, O.D., Koutsouradis, P., Chloros, G.D., Papanastasiou, I., Sarlikiotis, T., Kaspiris, A., Papagelopoulos, P.J., 2019. Bone tumours around the elbow: a rare entity. EFORT Open Rev. 4, 133-142.

14. Tunio, M.A., Alasiri, M., Ali, A.M., Alsaeed, E.F., Shuja, M., Fatani, H., 2013. Distal humerus as delayed site of metastasis from small cell carcinoma of gallbladder. Case. Rep. Gastrointest. Med. 2013, 946835.

15. Vaquero-Picado, A., Barco, R., Antuña, S.A., 2016. Lateral epicondylitis of the elbow. EFORT Open Rev. 1, 391-397.

16. Verhaar, J.A., 1994. Tennis elbow. Anatomical, epidemiological and therapeutic aspects. Int. Orthop. 18, 263-7.

17. Wang, C., Lin, N., 2015. Ewing's sarcoma of the ulna treated with sub-total resection and reconstruction using a non-vascularized, autogenous fibular graft and hernia mesh: A case report. Oncol. Lett. 10, 2067-2070. 